\title{
Defining the susceptibility of colorectal cancers to BH3-mimetic compounds
}

\author{
Ming-Jie Luo ${ }^{1,2}$, Michelle Palmieri ${ }^{1,3}$, Chris D. Riffkin ${ }^{1}$, Anuratha Sakthianandeswaren (1) ${ }^{1,3}$, Tirta Mario Djajawi ${ }^{1}$, \\ Yumiko Hirokawa', Victoria Shuttleworth ${ }^{1,10}$, David H. Segal ${ }^{1,3}$, Christine A. White $\mathbb{C}^{1,3}$, Duong Nhu1,3, \\ Guillaume Lessene ${ }^{1,3,4}$, Margaret Lee ${ }^{1,5,6,7}$, Peter Gibbs ${ }^{1,3,6}$, David C. S. Huang (1) ${ }^{1,3}$, Oliver M. Sieber ${ }^{1,3,8,9}$ and \\ Jia-nan Gong ${ }^{1,3}$
}

\begin{abstract}
Novel targets are required to improve the outcomes for patients with colorectal cancers. In this regard, the selective inhibitor of the pro-survival protein $B C L 2$, venetoclax, has proven highly effective in several hematological malignancies. In addition to $B C L 2$, potent and highly selective small molecule inhibitors of its relatives, $B C L x L$ and MCL1, are now available, prompting us to investigate the susceptibility of colorectal cancers to the inhibition of one or more of these pro-survival proteins. While targeting $B C L x L$, but not $B C L 2$ or $M C L 1$, on its own had some impact, most $(15 / 17)$ of the immortalized colorectal cancer cell lines studied were efficiently killed by the combined targeting of $\mathrm{BCLXL}$ and MCL1. Importantly, these in vitro findings were confirmed in a xenograft model and, interestingly, in all (5/ 5) patient derived tumor organoids evaluated. Our results lend strong support to the notion that BCLXL and MCL1 are highly promising targets for further evaluation in efforts to improve the treatment of colorectal cancers.
\end{abstract}

The direct induction of apoptosis by the BCL2-selective inhibitor, venetoclax (ABT-199), has led to significant progress in treating several blood cancers ${ }^{1}$. Whether BCL2, or its close relatives such as BCLxL or MCL1, is critical for maintaining the survival of solid cancers, including colorectal cancers (CRCs), is not fully defined. We addressed this question by testing immortalized human CRC cell lines in vitro and in vivo, importantly undertaking additional tests of the efficacy of such BH3mimetic drugs in patient-derived tumor organoids since these are most likely to inform us of the potential clinical utility of such drugs ${ }^{2}$.

Previous studies have implicated BCLxL and MCL1 in maintaining the survival of $\mathrm{CRCs}^{3-5}$, but their relative

\footnotetext{
Correspondence: David C. S. Huang (huang_d@wehi.edu.au) or

Oliver M. Sieber (sieber.o@wehi.edu.au) or Jia-nan Gong (gong.j@wehi.edu.au)

${ }^{1}$ The Walter and Eliza Hall Institute of Medical Research, Melbourne, VIC, Australia

${ }^{2}$ School of Medicine, Tsinghua University, Beijing, China

Full list of author information is available at the end of the article.

These authors contributed equally: Ming-Jie Luo, Michelle Palmieri
}

importance has not been clearly established. Thus, we independently tested a panel of 17 well-characterized human CRC cell lines with small molecules ${ }^{6}$ that selectively and potently inhibit BCL2 (venetoclax), BCLxL (A1331852), or MCL1 (S64845). Targeting just BCLxL could induce killing in $3 / 17$ lines, but inhibiting just MCL1 or BCL2 proved ineffective in any of the cell lines tested even after prolonged drug treatment (Fig. 1a, Supplementary Fig. 1).

This result, suggesting the primacy of BCLxL in CRC, prompted us to ask if MCL1 or BCL2 played secondary roles. Strikingly, 15/17 lines were susceptible when BCLxL and MCL1 were both targeted and co-inhibiting them killed as efficiently as combined treatment with all three BH3 mimetics. Thus, while BCLxL is the primary survival factor in CRC, MCL1 must play a secondary role and BCL2 is relatively unimportant. Of note, the killing induced by these $\mathrm{BH} 3$ mimetics was clearly through the specific induction of apoptosis it was fully abrogated in the absence of the apoptosis effectors BAX/BAK (Fig. 1b). 


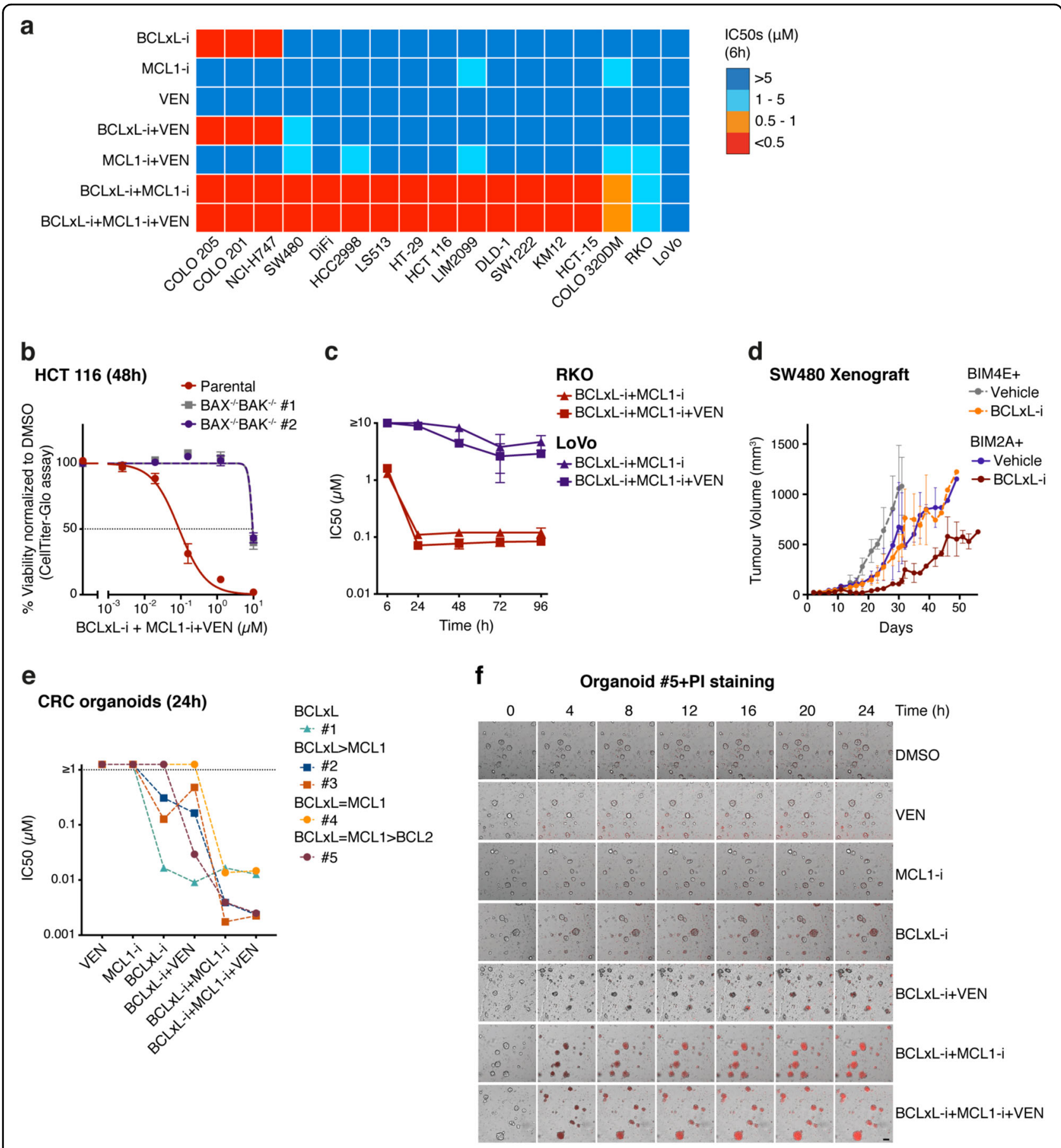

Fig. 1 (See legend on next page.)

Two of 17 lines (RKO and LoVo) remained viable even after $6 \mathrm{~h}$ exposure to the triple drug combination. RKO cells showed delayed responses (Fig. 1c), but in contrast, the LoVo cells were highly refractory even with prolonged treatment. In this regard, it is known that the LoVo cell line harbors inactivating mutations in $B A X^{7}$. We then sought to confirm the importance of BCLxL and MCL1 in vivo, by testing mice engrafted with the CRC cell line SW480. While modest suppression of tumor growth could be seen by targeting just BCLxL or MCL1, a deeper 
Fig. 1 Colorectal cancer cells are highly susceptible to the dual inhibition of BCLxL and MCL1. a In vitro sensitivity of a panel of colorectal cancer cell lines to the BH3-mimetic drugs. Seventeen CRC lines were treated with 0-10 $\mu \mathrm{M}$ of BH3 mimetics targeting BCLxL, MCL1, and BCL2, alone or in equimolar combinations (1:1 or 1:1:1). Cell viability was determined after $6 \mathrm{~h}$. A discrete heatmap representation of the mean $I C_{50}$ is shown; red represents potent killing $\left(I C_{50}<0.5 \mu \mathrm{M}\right)$, whereas blue indicates refractoriness $\left(I C_{50}>5 \mu \mathrm{M}\right)$. See Supplementary Table 1 for detailed information of the cell lines used. $\mathbf{b}$ Killing caused by the combined action of inhibitors targeting BCLXL, MCL1, and BCL2 is mediated by BAX and BAK. The viability of parental HCT 116 cells or a $B A X^{-1-} B A K^{-1-} H C T 116$ sub-clone was determined $48 \mathrm{~h}$ after the combined inhibition of BCLXL, MCL1, and BCL2. c LoVo cells are highly refractory to apoptosis induction. Sensitivity (mean $\mathrm{IC}_{50} \mathrm{~S} \pm \mathrm{SD}$ ) of RKO or LoVo cells treated with the indicated combinations of BH3 mimetics for 6-96 $\mathrm{h}$ is shown. $\mathbf{d}$ Expression of the MCL1-selective peptide ${ }^{10}$, BIM2A, enhances the in vivo suppression of tumor growth by the BCLxL inhibitor. The SW480 cells engineered to inducibly express BIM2A or BIM4E (inert control) were inoculated subcutaneously into immunodeficient NSG (NOD SCID IL-2R ${ }^{-1-}$ ) mice and treatment commenced 1 week later with $25 \mathrm{mg} / \mathrm{kg}$ of the BCLxL inhibitor A1331852 on weekdays for 2 weeks together with doxycycline-containing food to induce expression of BIM2A or BIM4E. Tumor sizes were monitored every 2-3 days and the data shown represent the mean tumor volumes \pm SD of three mice in each group. e Sensitivity of patient-derived tumor organoids to the BH3 mimetics. Patientderived tumor organoids $(n=5)$ were treated with $0-1.25 \mu \mathrm{M}$ of the indicated BH3-mimetic, alone, or in equimolar combinations (1:1 or 1:1:1). Cell viability was determined $24 \mathrm{~h}$ later and the mean $\mathrm{IC}_{50} \mathrm{~S}$ of two independent experiments are shown. The relative dependency of each organoid on $B C L x L, M C L 1$, and/or BCL2 is inferred. $\mathbf{f}$ Rapid induction of apoptosis by BH3-mimetic treatment. The viability of organoid \#5 treated with different BH3 mimetics (see e) was determined by PI (red) staining and imaged at indicated time points (see Supplementary Video 1). Scale bar $=100 \mu$ m. Cell viability in $\mathbf{a}-\mathbf{c}$, e was determined using CellTiter-Glo assays; data in $\mathbf{b}, \mathbf{c}$ represent the means \pm SD from three independent experiments. See Methods in Supplementary Material for experimental details.

impact was observed by co-targeting both of these prosurvival proteins (Fig. 1d).

Next, we tested whether this conclusion holds in patient-derived tumor organoids ${ }^{2}$. Remarkably, $3 / 5$ tumor organoids $(C R C \# 1-3)$ were variably sensitive to BCLxL inhibition alone (Fig. 1e). CRC\#1 relies predominantly on BCLxL, while MCL1 was also important in CRC\#2-4, and BCL2 contributed additionally in CRC\#5. Notably, marked killing of all five was achieved by combined inhibition of BCLxL, MCL1, and BCL2 (Fig. 1e and Supplementary Fig. 2a). The rapid killing of these organoids by these BH3 mimetics was confirmed by the killing kinetics with propidium iodide staining analyzed by microscopic imaging (Fig. 1f, Supplementary Fig. 2b, and Supplementary Video 1).

Our study and others ${ }^{3-5,8}$ pinpoint the importance of BCLxL and MCL1 for maintaining the survival of CRC cells. While the co-targeting of BCLxL and/or MCL1 may well be challenging because of on-target toxicities ${ }^{9}$, the action of the BH3 mimetics could be restricted to tumor cells with antibody-drug conjugates to preferentially target tumor tissues. Our findings will also be useful to develop rational combinations with standard-of-care agents, for example, combining BCLxL inhibition with cytotoxic agents that degrade MCL1, or, more generally, to evaluate BH3-mimetic therapy as sensitizers for agents used to treat CRC. Our study highlights the importance of further laboratory studies to investigate the full potential of BH3-mimetic drugs for treating CRCs, particularly for the large unmet need of patients who are fit for further therapies, but are refractory to all current standard-ofcare treatment approaches.

\section{Acknowledgements}

We thank Z. Xu, R. Thijssen, M.F. van Delft, and S. Huang for helpful discussions and suggestions. This work is supported by scholarships, fellowships, and grants from the Australian National Health and Medical Research Council
(NHMRC) (Research Fellowships 1136119 to O.M.S. and 1156024 to D.C.S.H.; Program Grant 1113133 to D.C.S.H.), Melbourne University (MIRS and MIFRS scholarships to T.M.D.), the China Scholarship Council (M.-J.L.), the Stafford Fox Medical Research Foundation (O.M.S.), and the Australian Cancer Research Foundation. This work was made possible through Victorian State Government Operational Infrastructure Support and Australian Government NHMRC Independent Research Institute Infrastructure Support Scheme (IRIISS).

\section{Author details}

${ }^{1}$ The Walter and Eliza Hall Institute of Medical Research, Melbourne, VIC, Australia. ${ }^{2}$ School of Medicine, Tsinghua University, Beijing, China.

${ }^{3}$ Departments of Medical Biology, University of Melbourne, Melbourne, VIC, Australia. ${ }^{4}$ Department of Pharmacology and Therapeutics, University of Melbourne, Melbourne, VIC, Australia. ${ }^{5}$ Department of Medicine (Melbourne Medical School - Western Precinct), University of Melbourne, Melbourne, VIC, Australia. ${ }^{6}$ Department of Medical Oncology, Western Health, Melbourne, VIC, Australia. ${ }^{7}$ Department of Medical Oncology, Eastern Health, Melbourne, VIC, Australia. ${ }^{8}$ Department of Surgery, University of Melbourne, Melbourne, VIC, Australia. ${ }^{9}$ Department of Biochemistry and Molecular Biology, Monash University, Melbourne, VIC, Australia. ${ }^{10}$ Present address: Institute of Cellular Medicine, Newcastle University Medical School, Newcastle upon Tyne, UK

\section{Conflict of interest}

M.P., C.D.R., A.S., T.M.D., Y.H., D.H.S., C.A.W., D.N., G.L., M.L., P.G., D.C.S.H., O.M.S., and J.-n.G. are employees of the Walter and Eliza Hall Institute, which receives milestone and royalty payments related to venetoclax. D.C.S.H. has received research funding from Genentech and Servier.

\section{Publisher's note}

Springer Nature remains neutral with regard to jurisdictional claims in published maps and institutional affiliations.

Supplementary Information accompanies this paper at (https://doi.org/ 10.1038/s41419-020-02815-0).

Received: 18 May 2020 Revised: 15 July 2020 Accepted: 16 July 2020 Published online: 10 September 2020

\footnotetext{
References

1. Roberts, A. W. et al. Targeting BCL2 with venetoclax in relapsed chronic lymphocytic leukemia. N. Engl. J. Med. 374, 311-322 (2016).

2. Schutgens, F. \& Clevers, H. Human organoids: tools for understanding biology and treating diseases. Annu. Rev. Pathol. 15, 211-234 (2020).
} 
3. Beroukhim, R. et al. The landscape of somatic copy-number alteration across human cancers. Nature 463, 899-905 (2010).

4. Faber, A. C. et al. mTOR inhibition specifically sensitizes colorectal cancers with KRAS or BRAF mutations to BCL-2/BCL-XL inhibition by suppressing MCL-1. Cancer Discov. 4, 42-52 (2014).

5. Zhang, $\mathrm{H}$. et al. Genomic analysis and selective small molecule inhibition identifies $B C L-X(L)$ as a critical survival factor in a subset of colorectal cancer. Mol. Cancer 14, 126 (2015).

6. Merino, D. et al. BH3-mimetic drugs: blazing the trail for new cancer medicines. Cancer Cell 34, 879-891 (2018).
7. Rampino, N. et al. Somatic frameshift mutations in the BAX gene in colon cancers of the microsatellite mutator phenotype. Science 275, 967-969 (1997).

8. Soderquist, R. S. et al. Systematic mapping of BCL-2 gene dependencies in cancer reveals molecular determinants of $\mathrm{BH} 3$ mimetic sensitivity. Nat. Commun. 9, 3513 (2018)

9. Mason, K. D. et al. Programmed anuclear cell death delimits platelet life span. Cell 128, 1173-1186 (2007).

10. Lee, E. F. et al. A novel $\mathrm{BH} 3$ ligand that selectively targets $\mathrm{Mcl}-1$ reveals that apoptosis can proceed without Mcl-1 degradation. J. Cell Biol. 180, 341-355 (2008). 